Exploiting Regional Amplitude Envelopes:

A Case Study for Earthquakes and Explosions in the Korean Peninsula

M. E. Pasyanos, W. R. Walter, K. M. Mayeda

January 9, 2012

Bulletin of the Seismological Society of America 
This document was prepared as an account of work sponsored by an agency of the United States government. Neither the United States government nor Lawrence Livermore National Security, LLC, nor any of their employees makes any warranty, expressed or implied, or assumes any legal liability or responsibility for the accuracy, completeness, or usefulness of any information, apparatus, product, or process disclosed, or represents that its use would not infringe privately owned rights. Reference herein to any specific commercial product, process, or service by trade name, trademark, manufacturer, or otherwise does not necessarily constitute or imply its endorsement, recommendation, or favoring by the United States government or Lawrence Livermore National Security, LLC. The views and opinions of authors expressed herein do not necessarily state or reflect those of the United States government or Lawrence Livermore National Security, LLC, and shall not be used for advertising or product endorsement purposes. 
January 6,2012

\title{
Exploiting Regional Amplitude Envelopes: A Case Study for Earthquakes and Explosions in the Korean Peninsula
}

\author{
Michael E. Pasyanos, William R. Walter \\ Lawrence Livermore National Laboratory, 7000 East Avenue, L-046, P.O. \\ Box 808, Livermore, California 94551
}

Kevin M. Mayeda

Berkeley Seismological Laboratory, 215 McCone Hall, University of California, Berkeley, Berkeley, California 94720-4760

Also at Weston Geophysical Corporation, 181 Bedford St., Ste. 1, Lexington, Massachusetts 02420.

\begin{abstract}
We introduce a new method to use regional amplitude envelopes for event analysis. Building on the success of the coda wave method, we construct synthetic template envelopes that attempt to fit the whole waveform that includes multiple direct phases and their coda over a wide frequency band. The method makes use of our understanding of earthquake and explosion source models, regional wave propagation, and the relation between direct amplitudes and coda. We demonstrate the power of the method by examining earthquakes and nuclear explosions in the Korean Peninsula at regional distance stations MDJ and TJN. In order to implement the method, however, we need to account for propagation through the use of an attenuation model for the region, which we have developed, along with an empirical correction to provide for unaccounted effects in the direct-to-coda transfer function. Under the assumption that the our explosion and attenuation models are correct, as well as the empirically-obtained transfer function, we find the 2006 DPRK test is consistent with a yield between 200 and 800 tons and a depth between 20 and 300 meters with our best fit at 500 tons at $100 \mathrm{~m}$ depth. Similarly the 2009 DPRK test is consistent with a yield range of 1-5 ktons and a depth range of 70 to $600 \mathrm{~m}$ with our best fit at $2 \mathrm{kton}$ and $200 \mathrm{~m}$.
\end{abstract}




\section{Introduction}

The coda wave method is a well-vetted empirical methodology to determine accurate magnitudes and explosive yields (Mayeda, 1993; Mayeda and Walter, 1996; Mayeda et al., 2003). The method works not by matching the amplitude of the direct arrival, which can often vary significantly, but by fitting the decaying amplitude of the coda from the local and regional phases. Normally, the method is implemented by focusing on a single regional phase type, usually an S-wave coda, such as Lg coda or Sn coda depending on the propagation characteristics of a region. More recently, some effort has been given to P-waves like Pn coda, but these phases are complicated by their shorter decay time before the arrival of interfering phases.

In many regions, however, either $\mathrm{Lg}$ or Sn can dominate depending on path, distance, and frequency (e.g. Pasyanos et al., 2011) making it hard to select one principal phase to calibrate and fit. Similarly, interference between P-waves and S-waves at short distances makes them hard to fit independently. We have also found that there is remarkable similarity in the attenuation characteristics of the coda and direct phases (Pasyanos et al., 2010), suggesting that we can utilize our extensive studies of attenuation of direct phase amplitudes to fit coda.

Here, we propose a new method to fit the envelope of the whole waveform at local and regional distances. By fitting the whole envelope, we include direct phases along with their associated coda, phases (both $\mathrm{P}$ and $\mathrm{S}$ ) that sample the earth in variety of ways, and over a wide frequency band. In a sense, we are trying to fit more and more of the whole waveform, by making fuller use of the amplitude time-bandwidth information available, without having to match the difficult to determine phase details by attempting to fit high-frequency waveforms swing-for-swing.

We will accomplish this by formulating the problem through the construction of synthetic template envelopes and comparing them to observed envelopes. We leverage existing work in coda calibration, direct phase attenuation, and source calibration to construct the full regional envelope for both explosion and earthquake sources for a range of distances, depths, emplacement conditions, etc. This method is appealing because it is based on physical models of the source spectrum, the scattering of direct phase energy into coda, and the propagation of regional phases, which should be applicable everywhere, although the propagation characteristics such as attenuation and site corrections need to be determined empirically.

After describing the method, we will apply the technique to a set of events in the Korean Peninsula (Figure 1) including the 2006 and 2009 presumed nuclear tests in the Democratic People's Republic of Korea (DPRK). We will demonstrate how the method can be used to improve our understanding of these explosions.

\section{Background}


As background, we will describe the coda wave method as a launching point for our method. As previously mentioned, the coda wave method is an empirical methodology to determine accurate magnitudes and explosive yields. Narrow-band timedomain envelopes are formed by the two horizontal components following the method of Mayeda et al. (2003). The coda envelopes of a particular phase (usually Lg or Sn) are then fit using the following form:

$$
A_{c}(f, t, r)=S(f) \cdot U(r, f) \cdot P(f) \cdot T(f) \cdot H(t-r / v(r)) \cdot(t-r / v(r))^{-\gamma(r)} \cdot \exp [b(r) \bullet(t-r / v(r))]
$$

where $\mathrm{S}(\mathrm{f})$ is the source amplitude, $\mathrm{U}(\mathrm{r}, \mathrm{f})$ is the propagation (geometrical spreading and attenuation), $\mathrm{P}(\mathrm{f})$ is the site response, $\mathrm{T}(\mathrm{f})$ is the direct phase-to-coda transfer function, $\mathrm{H}$ is the Heaviside step function, $t$ is the time in seconds from origin, $r$ is the epicentral distance in $\mathrm{km}, \mathrm{v}(\mathrm{r})$ is the velocity of the peak arrival in $\mathrm{km} / \mathrm{s}$, and $\gamma(\mathrm{r})$ and $\mathrm{b}(\mathrm{r})$ are coda shape parameters.

Calibrations of terms like propagation (attenuation, spreading, velocity) are performed for particular limited regions where they are applicable. Application of the method to broad regions has proven more challenging. Many of these same parameters, however, have already been calibrated for direct phase propagation. We would also like to generalize this method to fit the whole waveform, which will include all of the regional phases as well as background noise.

\section{Methodology}

We consider a less empirical, more physics-based method of fitting the complete regional amplitude envelope. The full regional waveform envelope can be described as sum of the noise and all of the regional phase amplitudes or:

$$
A_{\text {total }}=A_{\text {noise }}+A_{P n}+A_{P g}+A_{S n}+A_{L g}
$$

where $A_{\text {noise }}$ is the noise level in that frequency band, and $A_{P n}$ and $A_{S n}$ are zero at local distances where these phases are non-geometric. The regional phase terms $\left(\mathrm{A}_{\mathrm{Pn}}, \mathrm{A}_{\mathrm{Pg}}\right.$, $\mathrm{A}_{\mathrm{Sn}}, \mathrm{A}_{\mathrm{Lg}}$ ) include the direct phase and coda can be described as:

$$
A=A_{\text {direct }} \cdot H\left(t-t_{a}\right) \cdot\left(t-t_{a}\right)^{-\gamma(r)} \cdot \exp \left(b \cdot\left(t-t_{a}\right)\right)
$$

where $A_{\text {direct }}$ is the direct phase amplitude. This is the same form as equation (1) above, but with direct amplitudes (which include source, propagation, and site terms) replacing the first four terms used earlier. The only term not directly incorporated in the direct phase amplitudes is the transfer term $\mathrm{T}(\mathrm{f})$, which we will be considering more later. We have also replaced $r / v(r)$ with the arrival time $t_{a}$.

Conceptually, this is shown in Figure 2, where the envelopes will have a sawtooth pattern from the arrival and decay of local and regional phases. In order to populate equations (2) and (3) and construct synthetic template envelopes, we need the 
following five inputs: 1) Pre-event noise levels, 2) P-wave and S-wave source spectra, 3) Arrival times or velocities, 4) Propagation effects in terms of direct phase amplitude attenuation, geometrical spreading and site corrections, and 5) Coda decay parameters (b, $\gamma)$. They will be discussed in turn. We will be describing the method generally, although we will only apply it narrowly as described later on.

Noise

Background noise levels vary widely from station to station. We expect to see regional variations between noisy island and coastal stations to quiet inland stations far from the microseismic noise generated near coastlines. We also see variations due to siting conditions and distance from cultural noise. At some stations, we have also found large seasonal variations between summer and winter months, and diurnal variations due to cultural or wind-generated noise. While we would like to understand the noise variations for predictive purposes, the most direct way of including the noise is measuring the pre-event noise in every frequency band considered.

\section{Source Spectra}

We will be considering both earthquake and explosion source models. There are a variety of earthquake source spectra models. Here, we employ an MDAC source model (Walter and Taylor, 2001), which is a generalization of the Brune (1970) source spectrum that allows for arbitrary source scaling. Critical parameters for the model are the seismic moment Mo, which controls the long-period amplitude levels, and the corner frequency $\omega_{\mathrm{c}}$, which controls the amplitude roll-off with frequency.

For explosion spectra, the most-widely used is the Mueller-Murphy model (Mueller and Murphy, 1971), which describes P-wave spectra for a given yield, depth, and a number of source materials. A universally applicable S-wave spectrum for explosions has proven more elusive, and is still a topic of active research. Here, we employ the Fisk conjecture (Fisk, 2006) which specifies the corner frequency of S-waves relative to that of $\mathrm{P}$-waves is related to the ratio of the $\mathrm{P}$-wave and $\mathrm{S}$-wave velocities. For the low frequency level, we employ a similar ratio of wave velocities to the difference of the P-wave and S-wave amplitudes (Walter et al., 2007).

\section{Arrival Times}

Arrival times for the phases can most directly come from analyst picks. However, in the absence of picks or when poor signal-to-noise prevents this, it is useful to predict arrival times from appropriate velocity or travel time models. For example, we have used the regression of many regional phase picks to develop station-specific or regionallyappropriate phase velocity models. We can also use more sophisticated 2-D or 3-D models that can predict regional travel times like RSTT (Myers et al., 2010) and LLNLG3D (Simmons et al., 2011).

Propagation effects from Attenuation Tomography 
We have performed an attenuation tomography of the region using the amplitude inversion method of Pasyanos et al. (2009a) and generalized it to the multiphase amplitude tomography in Pasyanos et al. (2009b). In this method, we simultaneously invert the amplitudes of regional phases $\mathrm{Pn}, \mathrm{Pg}$, Sn, and Lg for the Qp and Qs structure of the crust and upper mantle. Amplitudes are modeled as a product of four terms: source term $\mathrm{S}$, geometrical spreading term $\mathrm{G}$, attenuation term $\mathrm{B}$, and site term $\mathrm{P}$.

$$
\mathrm{A}=\mathrm{S} * \mathrm{G} * \mathrm{~B} * \mathrm{P}
$$

True to their raypaths, $\mathrm{Pg}$ and $\mathrm{Lg}$ are modeled as propagating through the crust, while Pn and Sn propagate as mantle paths with source and station crustal legs. The amplitudes are then corrected for an assumed geometrical spreading and inverted for source, attenuation, and site terms.

Applying the tomography to East Asia forced us to focus on the complex tectonics of the region, including an imbedded region of oceanic crust and subducting slabs in the upper mantle. One thing we have done to improve the physics of the raypath is to introduce a variable crustal thickness into our inversion. This is particularly important in the Sea of Japan and Pacific Ocean, where the crustal thickness is less than $10 \mathrm{~km}$.

We perform the tomography in passbands of $0.5-1,1-2,2-4,4-6$, and 6-8 $\mathrm{Hz}$. Figure 3 shows path and attenuation results in East Asia in the 1-2 Hz passband. Notice the small number of Pg and Lg measurements (passing the signal-to-noise criteria) crossing the Sea of Japan, even though we have picked and measured hundreds of these paths (see Pn and Sn path maps). The tomographic inversion shows relatively simple crustal attenuation structure in the Korean Peninsula and the Yellow Sea, having lower Q in the Bohai Basin, but gets more complicated outside of this region, particularly to the east. We find low Q in the crust for the Sea of Japan. We also see complicated structure in the mantle that seems to be related to slab structure, but mainly for P-wave attenuation.

Coda parameters

The coda decay, or coda shape, parameters $b$ and $\gamma$ are functions of phase, distance, frequency, and region. As can be seen from equation (3), $\gamma$ controls the shape close to the direct phase, while b controls the decay at longer time intervals. Calibrations of these parameters is generally done for a single phase in a spatially limited region that is thought to have non-significantly-varying values. The variation with distance and frequency is achieved by having a distance dependent function for each frequency band, where the function is of the form:

$$
\begin{aligned}
& b(r)=b_{0}-b_{1} /\left(b_{2}+r\right) \\
& \gamma(r)=\gamma_{0}-\gamma_{1} /\left(\gamma_{2}+r\right)
\end{aligned}
$$


where $r$ again is epicentral distance. In this region, we use the coda shape parameters of Yoo et al. (2011), which were calibrated using Lg in the Korean Peninsula and are shown in Table 1. Values for the other phases are then scaled by their relative wavelengths.

\section{Event Analysis}

Using regional amplitude envelopes, we have examined four seismic events: the two announced nuclear explosions from the Democratic People's Republic of Korea (DPRK) and two shallow earthquakes in the vicinity of these tests (Figure 1). We have analyzed the recording of these events at station MDJ (Mudanjiang, Heilongjiang Province, China) and station TJN (Taejon, Republic of Korea). Epicentral distances for the events at MDJ range from 339 to $447 \mathrm{~km}$, while events at TJN range from 487 to 605 $\mathrm{km}$. The waveforms of the explosions do see relatively larger P-wave amplitudes and relatively smaller S-wave amplitudes relative to the nearby earthquakes (Figure 4).

We have first produced waveform envelopes for the two earthquakes in several narrow frequency bands (1-1.5, 2-3, 4-6, and 6-8 Hz). Envelopes are determined by removing the instrument response, transferring the trace to velocity, filtering to the desired passband using a Butterworth filter, and calculating an envelope using the square root of the sum of the squares of the original trace and its Hilbert transform. Envelopes in narrow frequency bands $(2-3 \mathrm{~Hz})$ are shown by the blue lines in Figure 5.

We have constructed synthetic waveform envelopes for the earthquakes using the procedure described. These are shown as the dashed green lines in the figure. The noise level in each frequency is estimated empirically and arrival times for each phase come from analyst picks. Direct amplitude levels of the four primary regional phases are determined by propagating source spectral amplitudes through the attenuation model described above for the appropriate frequency band. For example, we use the $2-4 \mathrm{~Hz}$ attenuation model for the $2-3 \mathrm{~Hz}$ envelope. We use an earthquake source with moment magnitudes of 3.90 and 3.85 for the two events. The moment magnitude values were determined from Lg coda using the coda wave method that has been empirically calibrated to be consistent with seismic moment values determined from regional and global moment tensor inversions.

Clearly, the observations are more complicated than the synthetic envelopes, although the shape of the function appears reasonable, especially for the closer, higher amplitude event. We can see from the earthquakes in Figure 5 that the envelopes predicted for earthquakes with appropriate moments seem to underpredict the overall amplitudes. We stated earlier that several factors could contribute to a non-unitary transfer function from direct-to-coda amplitudes, including differences in the observed data levels on the horizontal and vertical components. For example, using stations in California, we have found that this can often depending on the near-surface density and velocity structure of the recording station.

While this transfer function would normally be determined more systematically, here it will simply be determined empirically by comparing our observations to 
predictions for several earthquakes, then applying this to the nuclear explosions. We perform a grid search of $\mathrm{P}$-wave and $\mathrm{S}$-wave amplitudes for the two earthquakes and determine an average P-wave and S-wave amplification of 2 for station MDJ. The corrected amplitudes are shown as the solid green lines in Figure 5, bringing the synthetics in line with the observed envelopes. Similar analysis at station TJN finds that no amplification is needed for either $\mathrm{P}$ or $\mathrm{S}$ amplitudes.

We now consider the explosions. First, we try to fit the envelopes with an earthquake source with estimated moment magnitudes of 3.63 and 4.11 for the 2006 and 2009 tests. The corrected earthquake envelopes, illustrated with the green lines, fit the Swaves as expected, but clearly do not fit the P-waves. The misfit of the earthquake source is significantly higher for these events than those for the earthquakes, demonstrating the potential use of this method as a discriminant. Using an explosion source model for a reference event (Mueller-Murphy model, 1 kton event, $100 \mathrm{~m}$ depth, granite, Fisk conjecture) which is shown by a magenta line, we fit the overall shape of the observed amplitudes, although the 1 kton event appears too large for the 2006 explosion and too small for the 2009 explosion.

Amplitude levels are sensitive to both the size (yield) and depth of the explosion. Accordingly, we perform a grid search of 2006 and 2009 DPRK tests for variable yield and depth of burial (DOB) assuming the same source type and material. We perform a search ranging from 0.01 to $100 \mathrm{ktons}$ in yield and from 10 to $1000 \mathrm{~m}$ in depth at every first significant digit of 1, 2, and 5. Results are shown in Figure 6, along with a standard depth of burial estimated for Kazakhstan (Israelsson, 1994). For the 2006 event, a minimum misfit is found at a yield of 500 tons (200-800 tons) and a DOB of $100 \mathrm{~m} \mathrm{(20-}$ $300 \mathrm{~m}$ ). For the 2009 event, the minimum occurs at a yield $=2$ ktons $(1-5 \mathrm{ktons})$ and $\mathrm{DOB}=200 \mathrm{~m}(70-600 \mathrm{~m})$. These results are plotted as the red lines in Figure 5. Plotted along with the minimum are contours of misfit, including ones indicating an RMS of 5\% and $10 \%$ greater than the minimum, which may be taken as surrogates for the uncertainty range of the parameters.

Using broadband $\mathrm{P}$-wave spectral ratios of the two explosions at common regional stations, the study of Murphy et al. (2010) concludes that the best fit is a pair of tests of yield of 0.9 ktons at 200 meters and 4.6 ktons at 550 meters, which are shown as circles on Figure 6. In both cases, we find a smaller, shallower event. The ratios of the yields and depths of the two events are approximately the same. Our results are also consistent with the analysis reported by the Director of National Intelligence (DNI) for the 2006 test (http://www.dni.gov/announcements/20061016 release.pdf) which reports "Analysis of air samples collected on October 11, 2006 detected radioactive debris which confirms that North Korea conducted an underground nuclear explosion in the vicinity of P'unggye on October 9, 2006. The explosion yield was less than a kiloton." For the 2009 test, the DNI reports (http://www.dni.gov/announcements/20090615 release.pdf) "The U.S. Intelligence Community assesses that North Korea probably conducted an underground nuclear explosion in the vicinity of P'unggye on May 25, 2009. The explosion yield was approximately a few kilotons. Analysis of the event continues." 


\section{Conclusions}

We have developed a methodology to fit regional amplitude envelopes over a wide frequency band. This physics-based method uses appropriate earthquake and explosion source spectra, exploits the close relationship between direct phases and coda, and more accurate raypaths for the observed regional phases. While making use of many of the benefits of the coda wave method and calibrations of the coda decay parameters, we also take advantage of attenuation models for detailed propagation through the crust and upper mantle.

We applied the method to several events in the Korean Peninsula. We used the earthquakes to calibrate some of the unmodeled transfer function of the envelopes and then applied it to the 2006 and 2009 events. We can readily discriminate them as nonearthquake sources from their inability to fit earthquake source models. Using an explosion source model, we find explosion source parameters of yield and depth-ofburial, along with corresponding uncertainties. The values that we obtain for the depth and yield of the events depends critically on both the empirically-derived transfer functions as well as the explosion source model, particularly the S-wave spectrum which is not well-understood. However, our estimates using this method are, for the most part, consistent with estimates from other sources. In the future, we would like to better understand and account for the transfer function we empirically calibrated here, in order to apply the method to regions without prior calibration events.

\section{Data and Resources}

Station MDJ is part of the China Digital Seismograph Network (CDSN). Waveform data is available at the IRIS data center (http://www.iris.gov). Station TJN is part of the Ocean Hemisphere Project (OHP) network. Waveform data is available from the University of Tokyo Earthquake Research Institute (ERI) OHP DMC (http://ohpdmc.en.u-tokyo.ac.jp).

\section{Acknowledgments}

We thank Sean Ford and Eric Matzel for making many of the amplitude measurements that went into the attenuation tomography for the region. This work was prepared under the auspices of the U.S. Department of Energy by Lawrence Livermore National Laboratory (LLNL) under contract DE-AC52-07NA27344. This is LLNL contribution LLNL-JRNL-******.

\section{References}

Brune, J., (1970). Tectonic stress and the spectra from seismic shear waves earthquakes, J. Geophys. Res., 75, 4997-5009. 
Fisk, M.D. (2006). Source spectral modeling of regional P/S discriminants at nuclear test sites in China and the Former Soviet Union, Bull. Seism. Soc. Amer., 96, 23482367.

Gudmundsson, Ó., and M. Sambridge (1998), A regionalized upper mantle (RUM) seismic model, J. Geophys. Res., 103, 7121-7136, doi:10.1029/97JB02488.

Israelsson, H. (1994). Analysis of historical seismograms - root mean square Lg magnitudes, yields, and depth of explosions at the Semipalatinsk Test Range, Geophys. J. Int., 117, 591-609.

Mayeda, K. (1993). mb(Lg coda): A stable estimator of magnitude, Bull. Seism. Soc. Amer., 83, 851-861.

Mayeda, K. and W.R. Walter (1996). Moment, energy, stress drop, and source spectra of western U.S. earthquakes, J. Geophys. Res., 101, 11195-11208.

Mayeda, K., A. Hofstetter, J.L. O'Boyle, and W.R. Walter (2003). Stable and transportable regional magnitudes based on coda-derived moment-rate spectra. Bull. Seism. Soc. Amer., 93, 224-239.

Mueller, R.A. and J.R. Murphy (1971). Seismic characteristics of underground nuclear detonations: Part I. seismic spectrum scaling, Bull. Seism. Soc. Amer., 61, 16751692.

Murphy, J.R., B.C. Kohl, J.L. Stevens, T.J. Bennett, and H.G. Israelsson (2010). Exploitation of the IMS and other data for a comprehensive, advanced analysis of the North Korean nuclear tests, in Monitoring Research Review: Ground-Based Nuclear Explosion Monitoring Technologies, LA-UR-10-05578, 456-465.

Myers, S.C., M.L. Begnaud, S. Ballard, M.E. Pasyanos, W.S. Phillips, A.L. Ramirez, M.S. Antolik, K.D. Hutchenson, J.J. Dwyer, C.A. Rowe, and G.S. Wagner (2010). A crust and upper mantle model of Eurasia and North Africa for Pn travel time calculation, Bull. Seism. Soc. Amer., 100, 640-656, doi:10.1785/0120090198.

Pasyanos, M.E., E.M. Matzel, W.R. Walter, and A.J. Rodgers (2009a). Broad-band Lg attenuation modeling of the Middle East, Geophys. J. Int., 177, 1166-1176, doi:10.1111/j.1365-246X.2009.04128.x.

Pasyanos, M.E., W.R. Walter, and E.M. Matzel (2009b). A simultaneous multi-phase approach to determine P-wave and S-wave attenuation of the crust and upper mantle, Bull. Seism. Soc. Amer., 99-6, 3314-3325, DOI: 10.1785/0120090061.

Pasyanos, M.E., W.R. Walter, E. Matzel, S.R. Ford, R. Gök, and A.J. Rodgers (2010). Calibrations of attenuation structure in Eurasia to improve discrimination and 
yield, 2010 Monitoring Research Review: Ground-Based Nuclear Explosion Monitoring Technologies, LA-UR-10-05578, 466-474.

Pasyanos, M.E., W.R Walter, E.M. Matzel, R. Gök, D.A. Dodge, S.R. Ford, and A.J. Rodgers (2011). Seismic attenuation, event discrimination, magnitude and yield estimation, and capability analysis, 2011 Monitoring Research Review: GroundBased Nuclear Explosion Monitoring Technologies, LA-UR-11-04823, 535-543.

Simmons, N.A, S.C. Myers, and G. Johannesson (2011). Global-scale P-wave tomography optimized for prediction of teleseismic and regional travel times for Middle East events: 2. Tomographic inversion. J. Geophys. Res., 116, B04305, doi:10.1029/2010JB007969.

Walter, W.R., and S.R. Taylor (2001). A revised magnitude and distance amplitude correction (MDAC2) procedure for regional seismic discriminants: theory and testing at NTS, Lawrence Livermore National Laboratory, UCRL-ID-146882 www.llnl.gov/tid/lof/documents/pdf/240563.pdf

Walter, W.R., E.M. Matzel, M.E. Pasyanos, D.B. Harris, R. Gok, and S.R. Ford (2007). Empirical observations of earthquake-explosion discrimination using $\mathrm{P} / \mathrm{S}$ ratios and implications for the sources of explosion S-waves, 29th Monitoring Research Review: Ground-Based Nuclear Explosion Monitoring Technologies, Denver, Colorado, September 25-27, 2007.

Yoo, S.-H., J. Rhie, H. Choi, and K. Mayeda (2011). Coda-derived source parameters of earthquakes and their scaling relationships in the Korean Peninsula, Bull. Seism. Soc. Amer., 101, 2388-2398, doi: 10.1785/0120100318. 
Table 1.

Calibration Parameters for Coda Shape Parameters $\gamma$ and $b$ for 16 Narrowband Frequencies

\begin{tabular}{|c|c|c|c|c|c|c|}
\hline \multirow{2}{*}{$\begin{array}{l}\text { Frequency } \\
(\mathrm{Hz})\end{array}$} & \multicolumn{3}{|c|}{ Coda shape parameter $\gamma$} & \multicolumn{3}{|c|}{ Coda shape parameter b } \\
\hline & $\gamma 0$ & $\gamma 1$ & $\gamma 2$ & b0 & b1 & $\mathrm{b} 2$ \\
\hline $0.05-0.1$ & 0.6 & -40 & 1001 & $-1.470 \mathrm{e}-4$ & 0.0 & 0 \\
\hline $0.1-0.2$ & 0.3 & -20 & 31 & $-2.190 \mathrm{e}-4$ & 0.0 & 0 \\
\hline $0.2-0.3$ & 0.3 & -250 & 1001 & $-4.670 \mathrm{e}-4$ & 0.1 & 200 \\
\hline $0.3-0.5$ & 0.3 & -270 & 971 & $-1.115 \mathrm{e}-3$ & 0.1 & 190 \\
\hline $0.5-0.7$ & -0.2 & -820 & 701 & $-1.740 \mathrm{e}-3$ & 0.0 & 0 \\
\hline $0.7-1.0$ & 0.0 & -330 & 331 & $-2.396 \mathrm{e}-3$ & 0.0 & 0 \\
\hline $1.0-1.5$ & 0.2 & -270 & 451 & $-3.160 \mathrm{e}-3$ & 0.0 & 0 \\
\hline $1.5-2.0$ & 0.3 & -140 & 331 & $-4.315 \mathrm{e}-3$ & 0.0 & 0 \\
\hline $2.0-3.0$ & 0.3 & -320 & 1001 & $-5.484 \mathrm{e}-3$ & 0.0 & 0 \\
\hline $3.0-4.0$ & 0.0 & -690 & 971 & $-6.927 \mathrm{e}-3$ & 0.0 & 0 \\
\hline $4.0-6.0$ & 0.0 & -620 & 991 & $-8.677 \mathrm{e}-3$ & 0.0 & 0 \\
\hline $6.0-8.0$ & -0.1 & -640 & 971 & $-9.731 e-3$ & 0.3 & 190 \\
\hline $8.0-10.0$ & -0.1 & -700 & 1001 & $-1.155 \mathrm{e}-2$ & 0.1 & 270 \\
\hline $10.0-15.0$ & -0.1 & -590 & 921 & $-1.300 \mathrm{e}-2$ & 0.1 & 30 \\
\hline $15.0-20.0$ & 0.1 & -100 & 181 & $-1.539 \mathrm{e}-2$ & 0.2 & 160 \\
\hline $20.0-25.0$ & 0.1 & -50 & 71 & $-1.162 \mathrm{e}-2$ & 2.0 & 660 \\
\hline
\end{tabular}




\section{Figure captions}

Figure 1. Map of the Korean Peninsula and vicinity showing location of stations (yellow triangle), earthquakes (green circles), and explosions (red circles). The two explosions overlap each other. DPRK and RoK refer to the Democratic People's Republic of Korea (North Korea) and the Republic of Korea (South Korea), respectively.

Figure 2. Illustration of expected envelope shapes for regional events. The plot shows logarithmic velocity variation as a function of time and the sawtooth pattern comes from the arrival and decay of prominent regional phases.

Figure 3. a) Path map of $\mathrm{Pn}, \mathrm{Pg}, \mathrm{Sn}$, and $\mathrm{Lg}$ phases in the East Asia region surrounding the Korean Peninsula in the 1-2 Hz passband. Earthquakes are shown as circles and stations as yellow triangles. b) Attenuation results of the region showing Qp and Qs in the crust and the upper mantle. Mantle plots are overlain by contours of slab depth for the Kurile/Japan/Izu-Bonin (right) and Ryukyu (bottom center) slabs (Gudmundsson and Sambridge, 1998).

Figure 4. Waveforms of four events recorded at station MDJ (top) and TJN (bottom). Events are sorted by distance. Earthquake waveforms are shown in green and explosion waveforms are shown in red. All traces have been bandpass filtered between 0.5 and 8 $\mathrm{Hz}$.

Figure 5. Waveform envelopes for the two earthquakes and two explosions recorded at station MDJ in the 2-4 Hz passband. The y-axis shows log amplitude of velocity. The envelopes determined with data from the two horizontal components are shown in blue. Synthetic envelopes for the events are shown in green with dashed and solid lines showing uncorrected and corrected synthetics. Synthetic envelopes for a $1 \mathrm{kton}$ explosion at $100 \mathrm{~m}$ depth are shown in cyan, while envelopes for the best-fitting explosions are shown in red.

Figure 6. Misfit of the 2006 and 2009 events as a function of depth of burial and explosive yield assuming the Mueller-Murphy model, granite shot point and the Fisk conjecture. The minimum RMS is indicated with the star. Contours of 5\% and $10 \%$ above the minimum are drawn. The line shows a standard depth of burial of $90(\mathrm{~W})^{1 / 3}$ (Israelsson, 1994). Also shown on the plots (as circles) are the yield/depth values from the study of Murphy et al. (2010). 
Figure 1

Study Area and Events

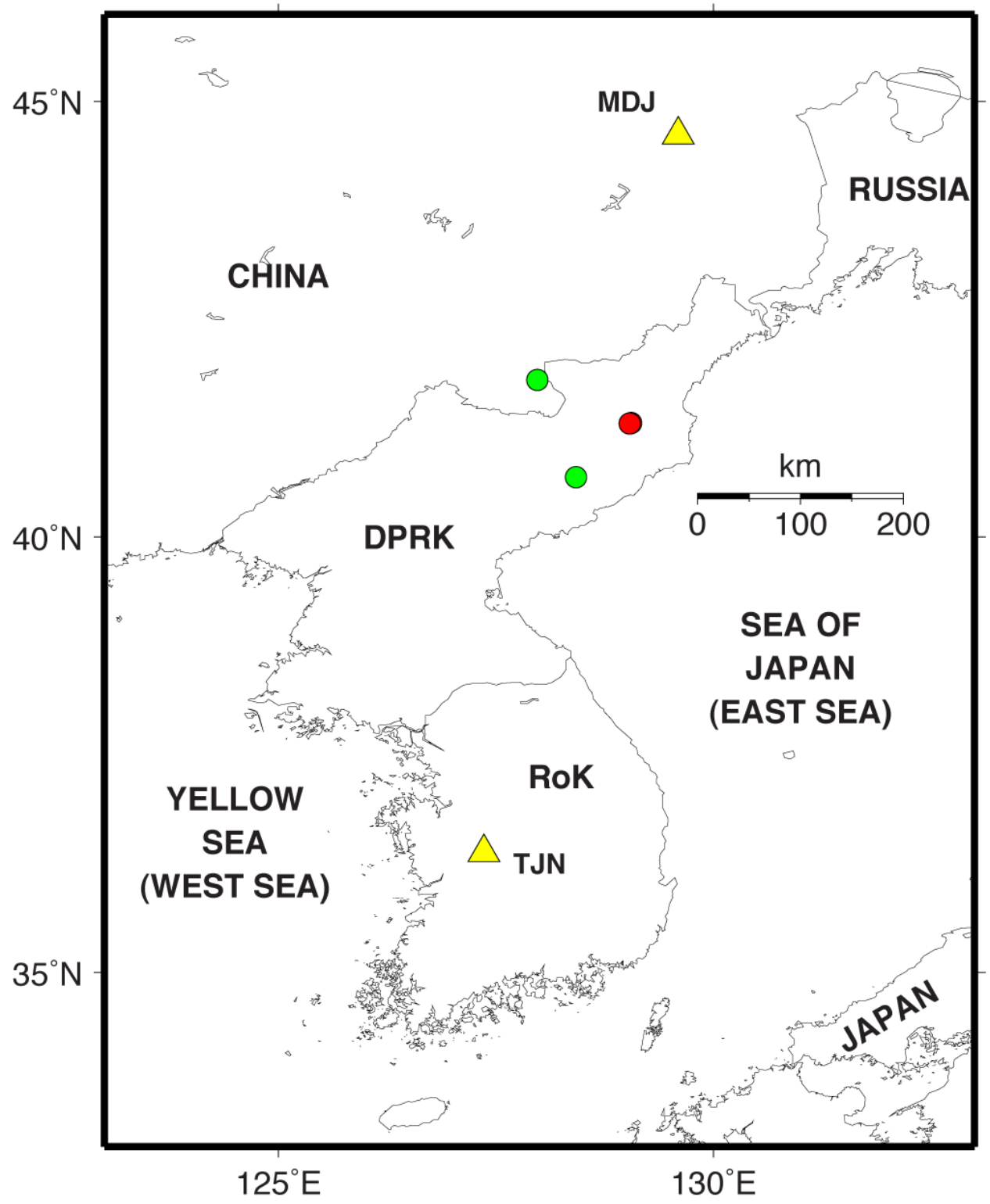


Figure 2

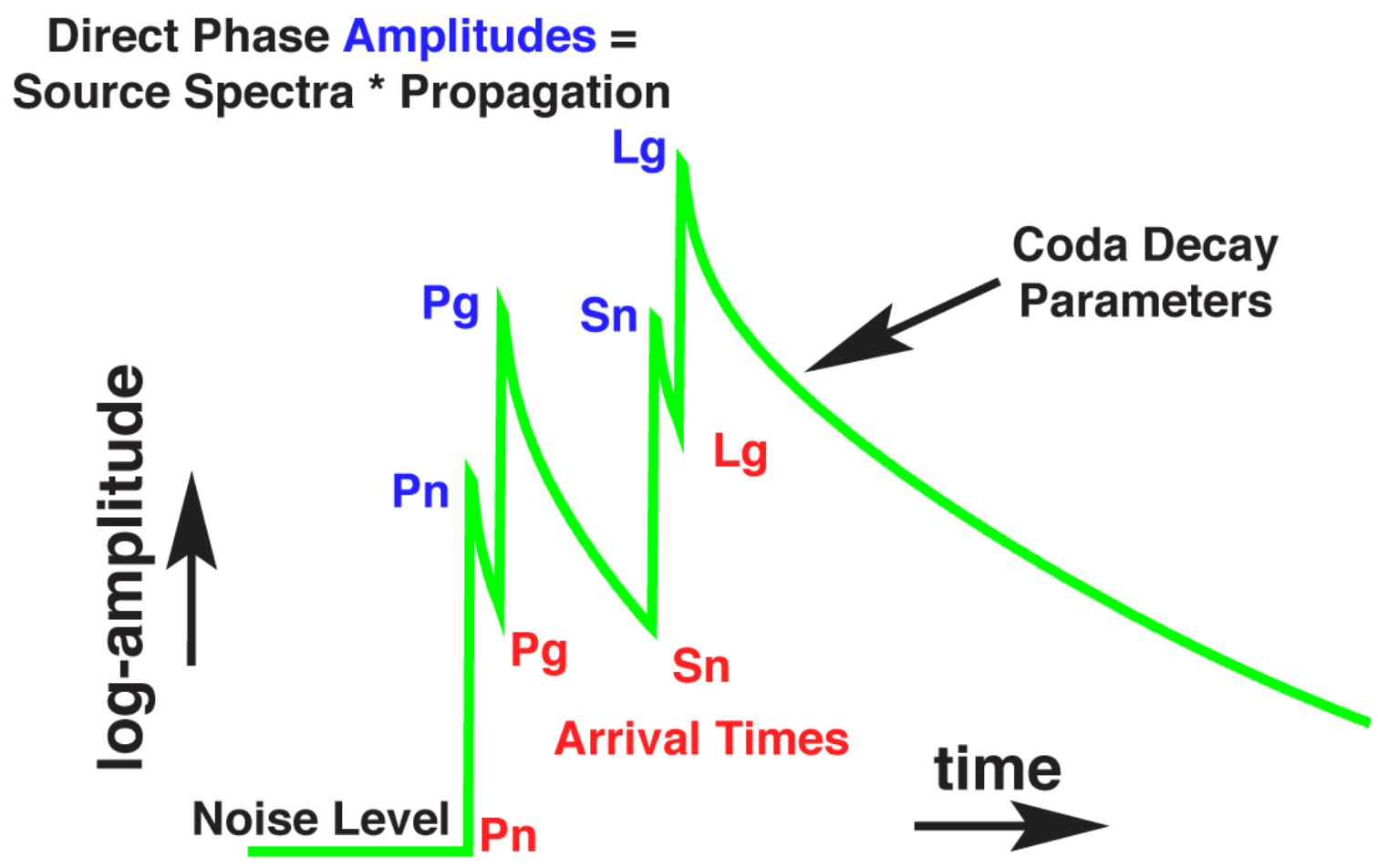


Figure 3

Pn Paths
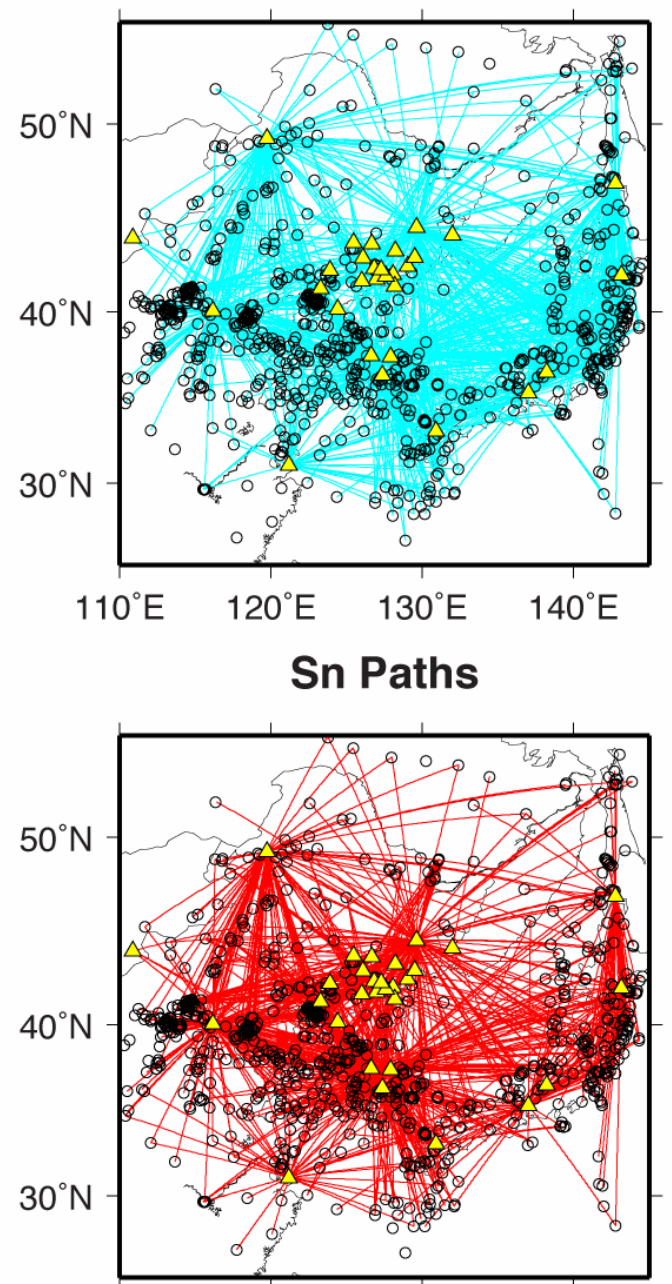

$110^{\circ} \mathrm{E} \quad 120^{\circ} \mathrm{E} \quad 130^{\circ} \mathrm{E} \quad 140^{\circ} \mathrm{E}$
Pg Paths
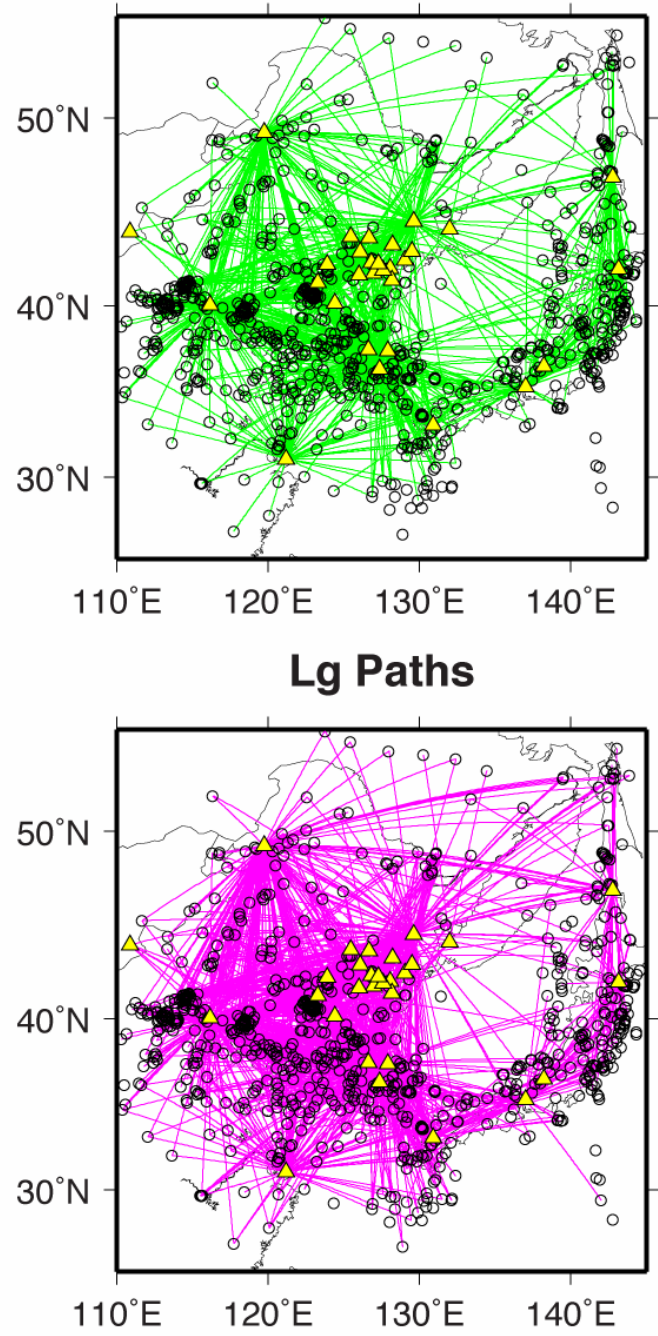
Figure 3 (cont)
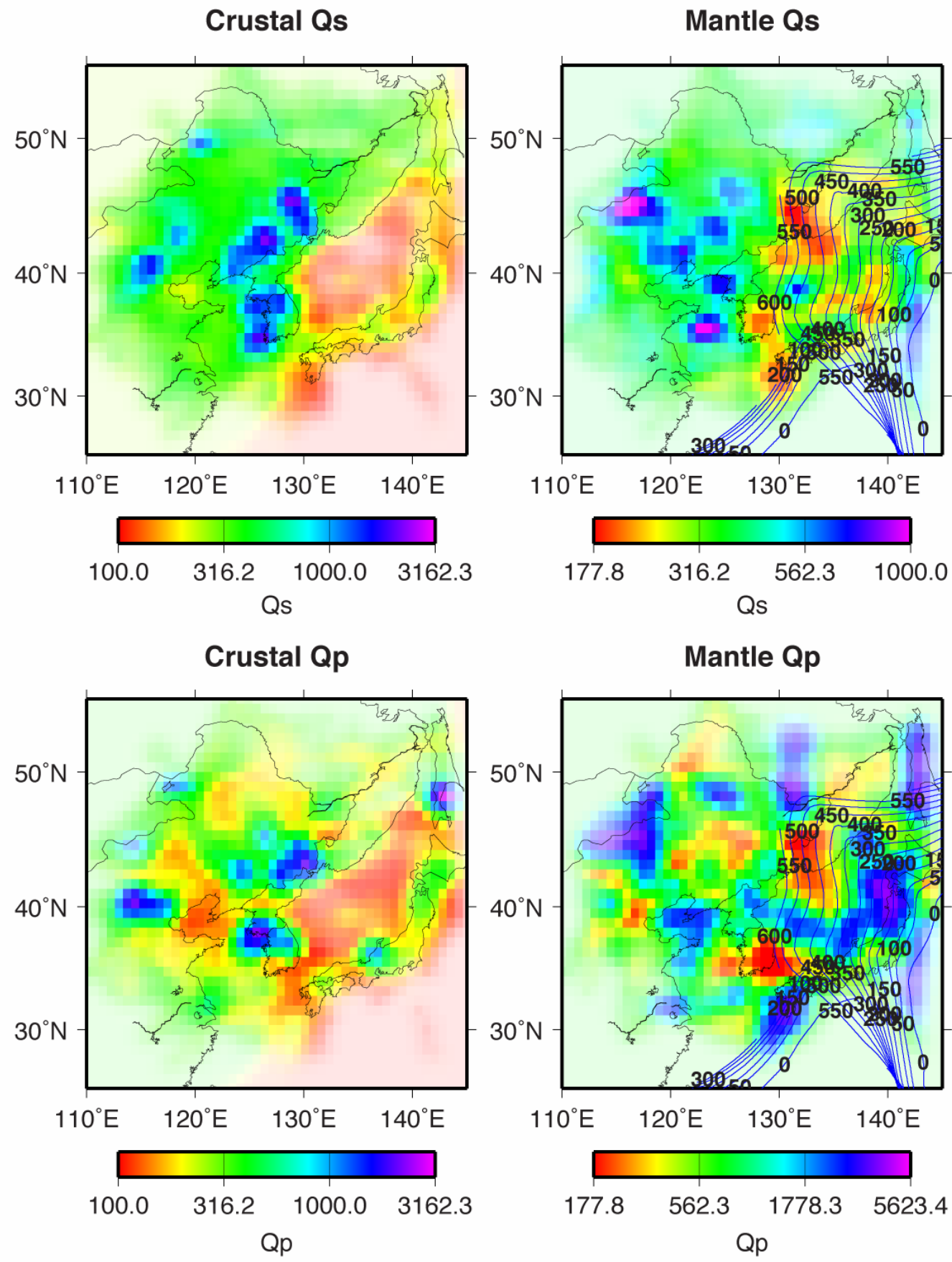
Figure 4
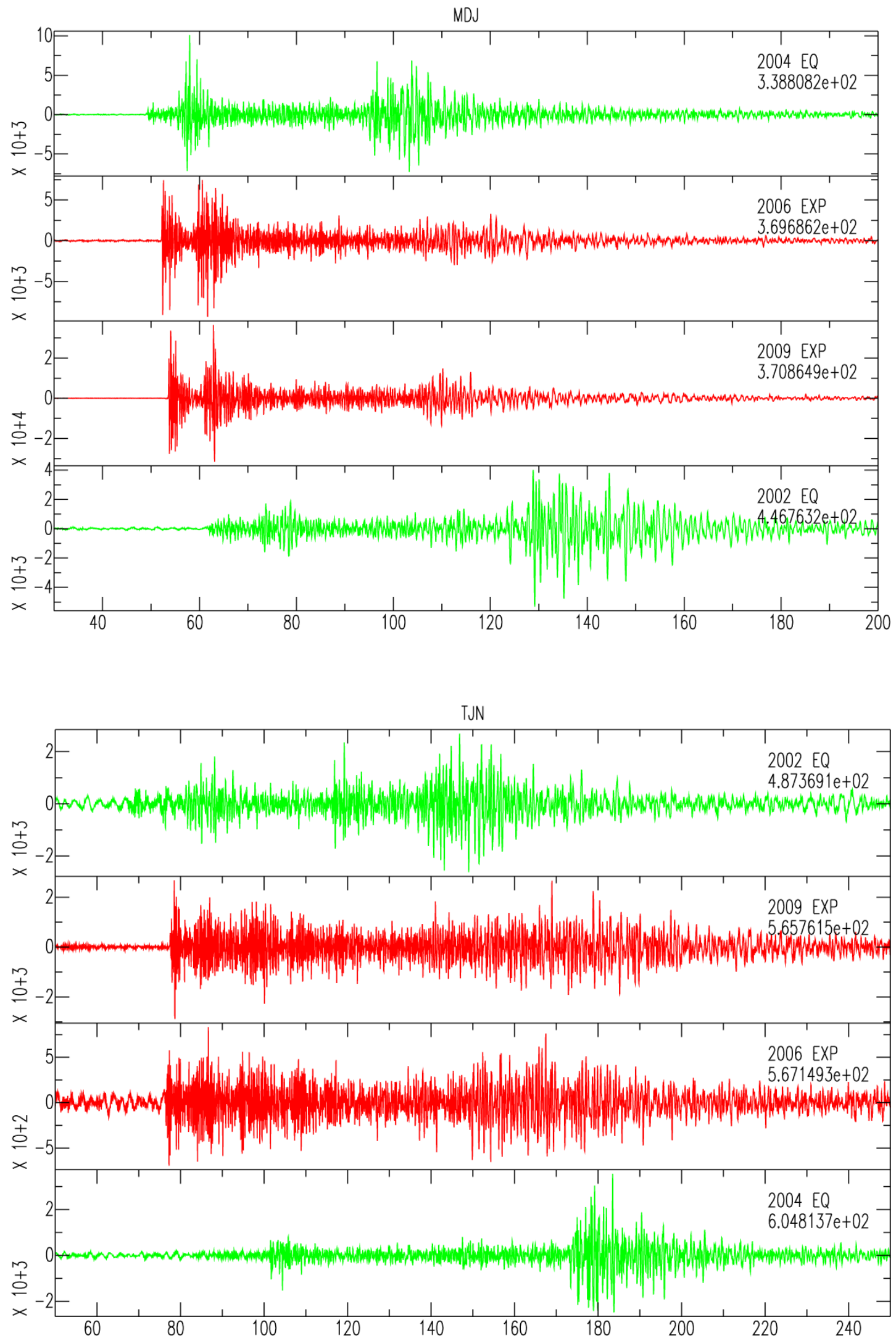
Figure 5
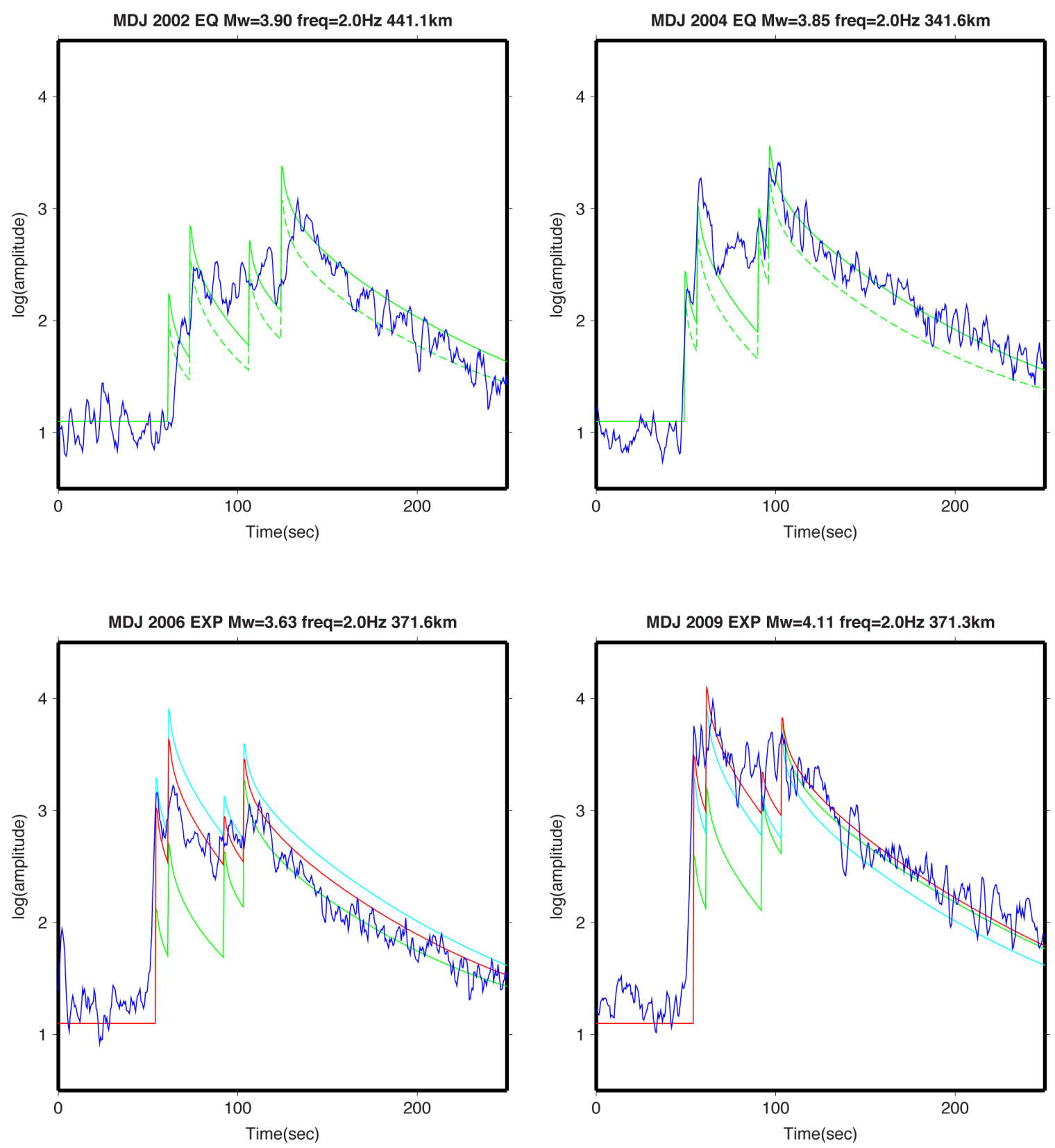
Figure 6
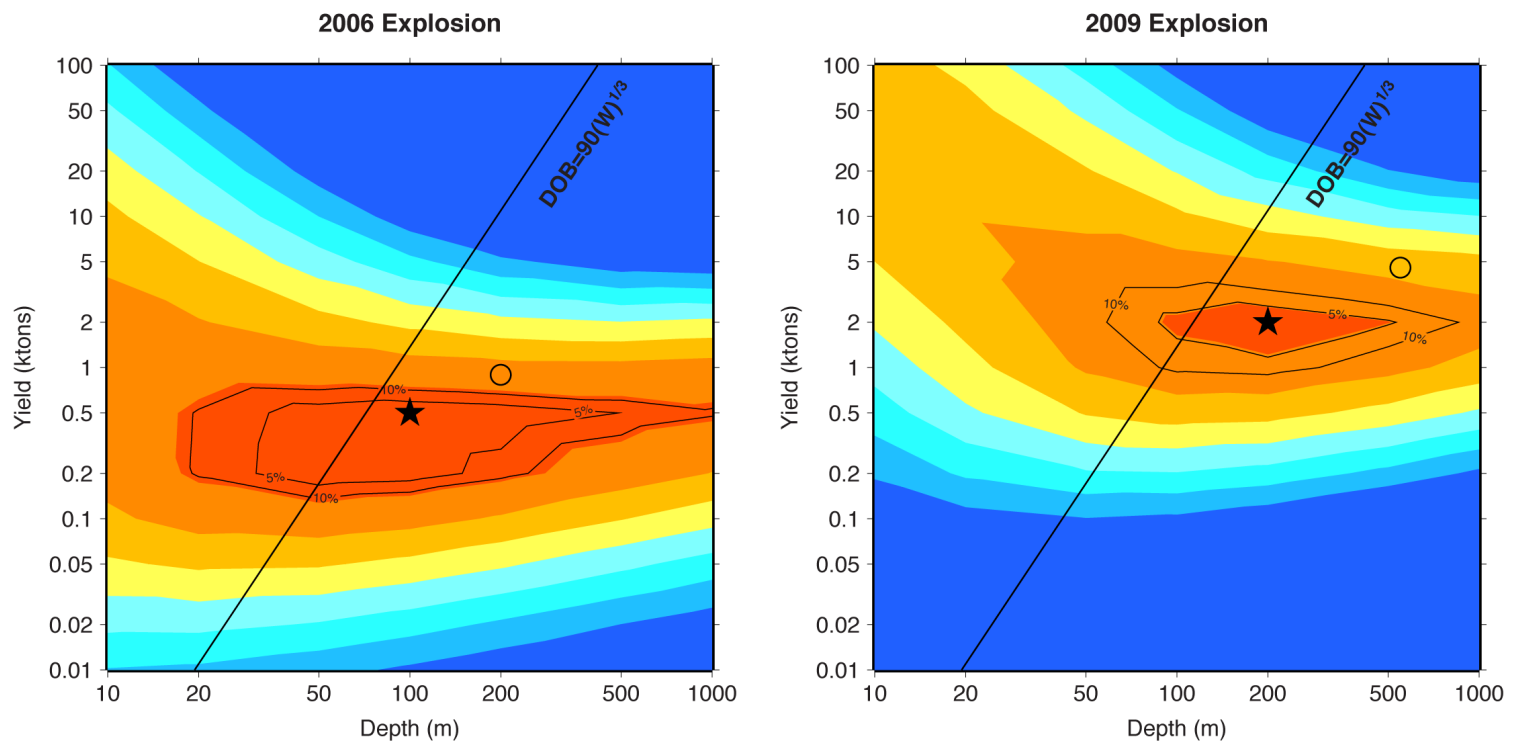

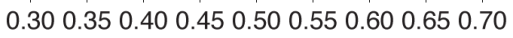

RMS Misfit 